\title{
As implicações do bullying na auto-estima de adolescentes
}

\author{
Cláudia de Moraes Bandeira \\ Claudio Simon Hutz
}

\begin{abstract}
Resumo
O objetivo do presente estudo foi investigar possíveis diferenças na autoestima de adolescentes envolvidos em bullying, enquanto agressores, vítimas, vítimas/agressores ou testemunhas, por sexo. Participaram 465 adolescentes, sendo $52,7 \%$ do sexo masculino. Os instrumentos utilizados foram um questionário sobre bullying e a Escala de Autoestima de Rosenberg. Os resultados apontaram para uma interação entre sexo e papéis de bullying em relação à autoestima. Testes Post Hoc demonstraram que, no grupo de vítimas/agressores, os meninos apresentaram média superior de autoestima em relação às meninas. Verificou-se que, em relação aos meninos, o grupo de testemunhas apresentou maior média de autoestima que o grupo de vítimas. Em relação às meninas, o grupo de agressoras apresentou média mais alta que o grupo de vítimas/ agressoras. Concluiu-se que o bullying apresenta diferentes implicações na autoestima de meninas e meninos envolvidos em diferentes papéis. Novos estudos para esclarecer algumas dessas questões são propostos.
\end{abstract}

Palavras-chave: Bullying, auto-estima, gênero.

\section{Implications of bullying in adolescents' self-esteem.}

\begin{abstract}
In this study we aim at investigating adolescents involved in bullying as victims, aggressor or victim/aggressor or even witness. We are interested in identifying the differences in their self-esteem according to gender. The participants, 465 adolescents (52.7\% males), completed the Rosenberg Self-Esteem Scale and a bullying questionnaire. Results showed an interaction between gender and the participants' roles in bullying in relation to self-esteem. Post-hoc analyses showed that in the victims/aggressors group, boys revealed higher self-esteem than the girls. Male witnesses presented higher self-esteem than victims. Female aggressors presented higher self-esteem than the victims/aggressors group. The results also show that bullying is a very common phenomenon which bring about different implications for girls and boys self-esteem. We propose new studies to clarify some of these issues.
\end{abstract}

Keywords: Bullying, self esteem, gender.

\section{Las consecuencias del bullying en la auto-estima de adolescentes}

\section{Resumen}

El objetivo del presente estudio fue investigar posibles diferencias por sexo en la auto-estima de adolescentes involucrados en bullying como agresores, víctimas, víctimas/agresores o testigos. Participaron 465 adolescentes, siendo $52,7 \%$ del sexo masculino. Los instrumentos utilizados fueron un cuestionario sobre bullying y la Escala de Auto-Estima de Rosenberg. Los resultados señalaron una interacción entre sexo y papeles de bullying en relación a la auto-estima. Tests Post Hoc demostraron que en el grupo de víctimas/agresores los chicos presentaron media superior de auto-estima en relación a las chicas. Se verificó que en relación a los chicos el grupo de testigos presentó media mayor de auto-estima que el grupo de víctimas. En relación a las chicas el grupo de agresoras presentó media más alta que el grupo de víctimas/agresoras. Se concluye que el bullying presenta diferentes consecuencias en la auto-estima de chicas y chicos involucrados en diferentes papeles. Se proponen nuevos estudios para aclarar algunos de eses aspectos.

Palabras clave: Bullying, auto-estima, género. 


\section{Introdução}

\section{Conceito de bullying}

O bullying constitui-se em uma subcategoria bem delimitada de agressão ou comportamento agressivo, caracterizado pela repetitividade e assimetria de forças (Olweus, 1993). É um comportamento agressivo e persistente com a intenção de causar dano físico ou moral em um ou mais estudantes que são mais fracos e incapazes de se defenderem (Olweus, 1993). A provocação é repetida e tem um caráter degradante e ofensivo, sendo mantida apesar da emissão de sinais claros de oposição e desagrado por parte do alvo. É intencional, não provocado pela vítima e pode ser considerado como uma forma de abuso, que pode ser tanto físico como psicológico. O bullying caracteriza-se por atos repetidos de opressão, tirania, agressão e dominação de pessoas ou grupos sobre outras pessoas ou grupos, subjugados pela força dos primeiros (Lopes, 2005).

No bullying, as agressões podem tomar a forma de abuso físico com a utilização de chutes, socos, pontapés, empurrões, roubo ou dano aos pertences. As agressões podem ser verbais, com a utilização de apelidos, insultos, comentários racistas, homofóbicos, de diferenças religiosas, físicas, econômico-sociais, culturais, morais e políticas (Ro$\mathrm{lim}, 2008)$. Podem também assumir uma forma mais indireta, como a exclusão social ou o isolamento (Olweus, 1993; Rigby, 1998). O bullying pode incluir chamar por nomes, debochar, chutar, bater, aterrorizar, ignorar e rejeitar, humilhar, intimidar, discriminar, entre outras ações agressivas (Lopes, 2005). Os ataques podem ocorrer também por vias eletrônicas, através de mensagens instantâneas, web site, salas de bate-papo ou torpedos. Este tipo de bullying tem sido referido como bullying eletrônico ou cyberbullying (Berger, 2007).

\section{Cenário do bullying}

O bullying tem sido considerado como um poderoso processo de controle social (Olweus, 1993). A vitimização pode ser considerada como um processo que ocorre na esfera coletiva, como um fenômeno social, em que a violência dos agressores é reforçada através da interação social entre os membros do grupo (Lisboa, 2005). O agressor tem sido considerado como um indivíduo procurando poder e liderança dentro do grupo de iguais (Olweus, 1993).

$\mathrm{O}$ agressor é aquela criança que age de forma agressiva contra um colega que é supostamente mais fraco, com a intenção de machucar, prejudicar, sem ter havido provocação por parte da vítima (Berger, 2007). O agressor, frequentemente, vê sua agressividade como qualidade, tem opiniões positivas sobre si mesmo e geralmente é bem aceito pelos colegas. Sente prazer e satisfação em dominar, controlar e causar dano nos outros e geralmente é mais forte que seu alvo (Lopes, 2005). Apresenta uma tendência maior para comportamentos de risco, como o consumo de tabaco, álcool ou outras drogas e porte de armas. Os agressores podem apresentar consolidação de seu papel de agressor com a continuidade deste ao longo da vida. Podem desenvolver sentimentos de culpa e vergonha pelos atos inadequados, isolamento ou exclusão social (Cantini, 2004). Rolim (2008) sustenta que o tipo pernicioso de agressão utilizada no bullying faz com que as vítimas sejam alvos que, por diferentes motivos, não conseguem se defender eficazmente das agressões. Isso faz com que os autores consigam solidificar suas posições na hierarquia do grupo a que pertencem ou também aumentem sua popularidade entre os colegas.

A vítima é o alvo do bullying e refere-se à criança que é repetidamente exposta a ações agressivas de outras crianças as quais têm a intenção de machucá-la e isso geralmente envolve diferença de força, tanto real, como percebida (Berger, 2007). As vítimas que são constantemente abusadas caracterizam-se por um comportamento social inibido, passivo ou submisso. Estes adolescentes costumam sentir vulnerabilidade, medo ou vergonha intensos e uma autoestima cada vez mais baixa, aumentando a probabilidade de vitimização continuada (Middelton-Moz \& Zawadski, 2007). As vítimas de bullying possuem até três vezes mais chances de sofrer com dores de cabeça e com dores abdominais, até cinco vezes mais chances de ter insônia e até duas vezes e meia mais chances de experimentar enurese noturna, quando comparadas às crianças que não são vítimas (Rolim, 2008). Segundo Lopes (2005), a redução da prevalência de bullying nas escolas pode ser uma medida de saúde publica altamente efetiva para o século XXI.

Algumas crianças são tanto vítimas como agressores e são denominadas de vítima/agressor. Estas crianças, provavelmente, apresentam uma combinação de baixa autoestima, atitudes agressivas e provocativas e prováveis alterações psicológicas, merecendo atenção especial. Podem ser depressivas, ansiosas, inseguras e inoportunas, procurando humilhar os colegas para encobrir suas limitações (Lopes, 2005). As vítimas/agressores têm uma maior probabilidade de apresentar sérios problemas de comportamento externalizado e são, em grande frequência, maltratadas por seus colegas. Experienciam dificuldades com o comportamento impulsivo, reatividade emocional e hiperatividade. Diferenciam-se dos alvos típicos por serem impopulares e pelo alto índice de rejeição entre seus colegas (Robin, Toblina, Schwartza, Gormanb, \& Abou-ezzeddinea, 2005).

O grupo de vítimas/agressores apresenta os maiores números de problemas de conduta, problemas na escola, problemas com o grupo de iguais, sintomas psicossomáticos e psicológicos, maiores encaminhamentos aos serviços psiquiátricos e uma maior probabilidade de persistência no seu envolvimento em bullying (Liang, Flisher, \& Lombard, 2007). As vítimas/agressores apresentam certas características como sintomas de depressão, ansiedade e outras formas de estresse internalizado. Alguns pesquisadores apresentam a hipótese de que o comportamento agressivo destas crianças reflete um estado de pobreza em modular a raiva e a irritabilidade maior do que a capacidade de utilização de estratégias sociais com um objetivo orientado (Robin e cols., 2005). Liang e cols. (2007) afirmam que, juntamente com o grupo de 
agressores, as vítimas/agressores estão mais suscetíveis ao uso excessivo de cigarros, álcool e outras substâncias. Este grupo apresenta o risco mais elevado para apresentar severas ideações suicidas. Apresentam risco aumentado para vários tipos de comportamento de risco, violência e comportamento antissocial, quando comparadas a crianças que não estão envolvidas em bullying (Liang e cols., 2007).

Em se tratando de vítimas/agressores, é importante fazer distinção entre comportamento agressivo proativo e reativo. O comportamento agressivo proativo envolve tentativas de influenciar o outro através de meios aversivos, em uma situação que não foi provocada (Gini \& Pozzoli, 2006). É um comportamento voluntário, deliberado e influenciado por reforços externos (Lisboa, 2005). Este é o tipo de agressão utilizada pelos agressores típicos. Já o comportamento agressivo reativo é um ato impulsivo em resposta a uma provocação ou ameaça percebida (Gini \& Pozzoli, 2006) e consiste em uma resposta defensiva de raiva (Lisboa, 2005). Este é o tipo de agressão utilizada pelas vítimas/agressores.

Berger (2007) afirma que a maior parte dos alunos não se envolve diretamente em atos de bullying e geralmente se cala por medo de ser a próxima vítima, por não saber como agir e por não acreditar nas atitudes da escola. Grande parte das testemunhas sente simpatia pelas vítimas, tende a não culpá-los pelo ocorrido, condena o comportamento dos agressores e deseja que os professores intervenham efetivamente (Lopes, 2005). Garandeau e Cillessen (2006) sustentam que, quando uma criança ou adolescente testemunha um colega sendo vitimizado por outro, o seu comportamento não será neutro. Poderá escolher o lado da vítima e se juntar ativamente ao bullying ou se manter passivo. Entretanto, é importante ressaltar que ser passivo não é o mesmo que ser neutro. Na realidade, a atitude passiva reforça a agressão, por mostrar ao agressor que nada irá interromper a atividade, deixando-o livre para realizar sua ação (Garandeau \& Cillessen, 2006).

Segundo Cantini (2004), é possível identificar alguns fatores de risco que podem estar associados à ocorrência do bullying, como fatores da personalidade, autoestima, dificuldades nas relações sociais, ser vitimizado na escola ou fora dela, violência na escola ou fora dela, violência na comunidade, desajustes familiares, práticas educativas parentais, contexto escolar, alienação escolar, violência na mídia e percepção do problema.

\section{Autoestima}

O conceito de autoestima tem sido estudado e considerado como um importante indicador de saúde mental na adolescência. Existe uma correlação entre autoestima, rendimento escolar e aprovação social, e tal correlação é virtualmente generalizável a todos os grupos étnicos e culturais (Steinberg, 1999). A autoestima é talvez a variável mais crítica que afeta a participação exitosa de um adolescente com outros em um projeto. Os adolescentes com baixa autoestima desenvolvem mecanismos que provavelmente distorcem a comunicação de seus pensamentos e sentimentos e dificultam a integração grupal (Coopersmith, 1989; Rosenberg, 1989). Um bom grau de autoestima é crítico para o bom funcionamento do adolescente, uma vez que ela ajuda os adolescentes a acreditarem e confiarem neles mesmos. A autoestima também afeta o adolescente na forma de lidar com o ambiente. Crianças e adolescentes com boa autoestima persistem mais e fazem mais progressos diante de tarefas difíceis que aqueles com uma baixa autoestima. A posição que as crianças e os adolescentes ocupam entre seus pares é extremamente importante, uma vez que a autoestima é uma função deste status dentro do grupo. As crianças cujos pares não gostam dela tem menos oportunidades de desenvolver suas habilidades sociais (Steinberg, 1999). A autoestima está relacionada à saúde mental e ao bem-estar psicológico e sua carência está relacionada a certos fenômenos mentais negativos como depressão e suicídio. Para Rosenberg (1989), pessoas com baixa autoestima engajam-se em comportamentos delinquentes como uma forma de retaliação contra a sociedade que desdenha deles e também como uma forma de obter autoestima.

Autoestima é uma avaliação que o indivíduo efetua e comumente mantém em relação a si mesmo. Expressa um sentimento ou uma atitude de aprovação ou de repulsa por si mesmo e refere-se ao quanto um sujeito considera-se capaz, significativo, bem sucedido e valioso (Coopersmith, 1989; Rosenberg, 1989). Pode ser entendida como um juízo pessoal de valor, externado nas atitudes que o indivíduo tem para consigo mesmo e nas crenças pessoais sobre suas habilidades, capacidades, relacionamentos sociais e acontecimentos futuros (Coopersmith, 1989; Heatherton \& Wyland, 2003). A autoestima diz respeito à forma como o indivíduo elege suas metas, aceita a si mesmo, valoriza o outro e estabelece suas expectativas e projetos, sendo que o ponto fundamental da autoestima é o aspecto valorativo (Coopersmith, 1989). A percepção que o indivíduo tem do seu próprio valor e a avaliação que faz de si mesmo em termos de competência constituem os pilares fundamentais da autoestima. Esta se constitui em uma experiência subjetiva, acessível às pessoas através de relatos verbais e comportamentos observáveis (Coopersmith, 1989).

Muitas das mais populares teorias sobre autoestima estão baseadas na teoria denominada The looking-glass self (Heatherton \& Wyland, 2003). Esta teoria, proposta por Charles Horton Cooley no início do século $X X$, sustenta que o indivíduo e a sociedade não existem separadamente, mas sim que um é produto do outro. Da mesma forma como nós descobrimos nossa aparência através do nosso reflexo no espelho, aprendemos sobre nossa personalidade olhando a reação dos outros. Se várias pessoas nos rodeiam, acreditamos que somos populares. Se as pessoas riem de nossas piadas, acreditamos que somos divertidos, engraçados. Em outras palavras, a forma como nós vemos a nós mesmos é fortemente influenciada pela maneira como os outros nos veem. Heatherton e Wyland (2003) afirmam que alguns estudos sugerem que meninos e meninas divergem na origem da autoestima, sendo que a autoestima das meninas é mais influenciada pelos relacionamentos, enquanto a autoestima 
dos meninos é mais influenciada pelo sucesso dos seus objetivos.

Existem controvérsias quanto à conceitualização da autoestima como um traço estável da personalidade ou como um estado especificamente ligado ao contexto (Heatherton \& Wyland, 2003). Algumas teorias apontam para autoestima como sendo um traço relativamente estável da personalidade do indivíduo. Dentro desta perspectiva, a autoestima pode ser considerada como estável, pois é construída vagarosamente ao longo do tempo, através de experiências pessoais bem sucedidas, sendo valorizada continuamente por pessoas significantes. Outras teorias apontam para uma classificação variável, com a asseveração de que a autoestima pode ser momentaneamente manipulada ou afetada. Entretanto, uma visão subsequente sustenta que a autoestima pode ser considerada tanto como um estado quanto como um traço (Heatherton \& Wyland, 2003). Rosenberg (1989) sugere que o julgamento pessoal é formado desde a infância. Embora no início da adolescência os indivíduos sejam suscetíveis à maior inconstância dos sentimentos sobre si, a autoestima tende à estabilidade ao longo do ciclo vital. Os achados de Rigby e Cox (1996), em seus estudos sobre a relação entre bullying e autoestima, apontam para uma relação entre baixa autoestima e bullying em meninas, mas não encontraram a mesma relação entre os meninos. Assim, o fenômeno de bullying deve ter diferentes implicações na autoestima dos adolescentes do sexo masculino e do sexo feminino.

\section{Diferenças de gênero}

Pesquisas em desenvolvimento de gênero mostram que meninas e meninos diferem na apresentação de vários problemas de desenvolvimento. Sexo e gênero são importantes fontes de variabilidade no comportamento das crianças (Bell, Foster, \& Mash, 2005). Apenas mostrar que meninas e meninos diferem não é o suficiente. Existe a necessidade de investigar como as experiências de socialização, variáveis biológicas e o contexto ambiental ou cultural interagem com o passar do tempo para produzir diferentes modelos de ajustamento para meninas e meninos.

Por muito tempo, os pesquisadores de bullying detiveram-se em estudar apenas os meninos, pois consideravam que este fenômeno ocorria com muito mais frequência nos indivíduos do sexo masculino (Berger, 2007). Mais recentemente, reconheceram-no também como um problema das meninas, mas, provavelmente, com uma apresentação única. Olweus (1993) acreditava que o bullying ocorria com pouca frequência nas meninas. A forma como o bullying apresenta-se nas meninas é geralmente despercebida, como se elas não fossem suspeitas de comportamento agressivo ou bullying da mesma forma que os meninos (Vail, 2002). Este dado é reforçado por Lisboa (2005), que identifica que os meninos são classificados pelos seus colegas como agressores e como vítimas/agressores com uma frequência maior do que as meninas. Para Liang e cols. (2007), a agressividade e a vitimização são de ocorrência mais comum entre os meninos. Já Gini e Pozzoli (2006) afirmam que a diferença entre meninos e meninas está no tipo de agressão utilizada e não na incidência de agressão nos subgrupos de meninos e meninas.

Algumas pesquisas apontam diferenças entre meninas e meninos em relação ao bullying, visto que comumente as meninas identificam-se mais como vítimas e testemunhas e os meninos mais como agressores e vítimas/agressores (Bandeira, 2009). As meninas geralmente expressam atitudes mais positivas em relação às vítimas, são mais empáticas e dão mais suporte que os meninos (Gini \& Pozzoli, 2006). Os meninos tendem a utilizar a agressão física como empurrões, chutes e socos. Já as meninas utilizam formas mais indiretas de bullying, como agressão verbal, insulto, mentira e fofoca (Bandeira, 2009). Nas adolescentes, em particular, é comum o uso de apelidos e fofocas (Vail, 2002). Os meninos afirmam que são mais agredidos por outros meninos, enquanto as meninas afirmam que são agredidas principalmente por outras meninas. Meninas e meninos também diferem na maneira como percebem e nas suas atitudes em relação ao bullying (Bandeira, 2009).

Crick e Grotpeter (1995) propuseram o termo agressividade relacional para denominar as ações cometidas pelas meninas, nas quais as interações sociais são manipuladas para causar prejuízo no relacionamento entre os pares. Isso envolve ameaças de expulsão do grupo, exclusão proposital e comentários prejudiciais a respeito de alguém com o fim de causar a rejeição do grupo de pares. Os autores acima citados acreditam que as meninas utilizam mais esse tipo de abuso que os meninos, uma vez que isso prejudica o que mais importa para elas, o relacionamento entre o mesmo gênero. Segundo Lisboa (2005), as relações diádicas e íntimas parecem ser mais importantes para as meninas que para os meninos. As meninas tendem a se importar mais com o retorno dos pares para formar seu autovalor, o que torna as adolescentes mais suscetíveis aos comentários em relação à sua aparência física (Crick \& Grotpeter, 1995). Conforme Lisboa (2005), é permitido socialmente às meninas manter relações de amizades íntimas e próximas com um par do mesmo sexo. Já os meninos são vulneráveis a preconceitos, podendo, por isso, tornarem-se vítimas.

\section{Método}

O presente estudo seguiu um delineamento do tipo correlacional de corte transversal. Este estudo contou com 465 adolescentes, estudantes de quartas a oitavas séries do ensino fundamental de três escolas (duas públicas e uma privada) da cidade de Porto Alegre, RS. Dentre estas crianças, 220 pertenciam ao sexo feminino $(47,3 \%)$ e 245 , ao sexo masculino (52,7\%). A idade dos participantes variou entre nove e 18 anos $(M=13,4 ; d p=1,47)$. A amostra foi de conveniência e a participação dos alunos foi voluntária. Este estudo foi aprovado pelo Comitê de Ética em Pesquisa do Instituto de Psicologia da Universidade Federal do Rio Grande do Sul. 


\section{Instrumentos}

Os instrumentos utilizados foram um questionário sobre bullying e a Escala de Autoestima de Rosenberg. O questionário sobre bullying contou com 15 questões de múltipla escolha em que os adolescentes puderam se identificar como participantes, ou não, de bullying. Algumas das questões possibilitaram a escolha de mais de uma opção, permitindo sobreposições de algumas categorias a priori. $\mathrm{O}$ questionário possibilitou identificar os adolescentes enquanto vítimas, agressores, vítimas/agressores e testemunhas.

A Escala de Autoestima de Rosenberg (1989) é uma medida unidimensional que avalia a autoestima global. É constituída por dez afirmações relacionadas ao conjunto de sentimentos de autoestima e autoaceitação. Os itens são respondidos em uma escala de quatro pontos variando entre concordo totalmente, concordo, discordo e discordo totalmente. Neste estudo, foi utilizada a versão adaptada para o português por Hutz (2000), que manteve a avaliação como uma medida unidimensional com características psicométricas equivalentes às do instrumento original. No presente estudo, o instrumento manteve seus níveis de fidedignidade.

\section{Procedimentos}

Inicialmente, foi encaminhado um termo de consentimento livre e esclarecido para assinatura dos pais ou responsáveis de todos os participantes. Os questionários foram aplicados coletivamente em sala de aula, em dias e horários designados previamente. A coleta dos dados teve a duração de 50 minutos. Antes de iniciar a aplicação dos questionários, a pesquisadora realizou um rapport, no qual explicou aos alunos os objetivos da pesquisa e o significado do termo bullying, citando alguns exemplos, sempre utilizando uma linguagem apropriada para a faixa etária dos participantes. Neste rapport, a pesquisadora explicou a diferença entre bullying e agressões ocasionais, explicando as características peculiares do bullying. Os adolescentes foram informados que sua participação era voluntária e que poderiam interrompê-la a qualquer momento sem nenhuma penalidade. Também foram informados sobre o sigilo e a confidencialidade dos dados e de que não deviam se identificar nos questionários. Os únicos dados pessoais que foram coletados foram idade, sexo, com quem reside e o número de irmãos.

\section{Resultados}

Inicialmente, foi feita uma verificação das características psicométricas da escala de autoestima na amostra do estudo. Verificou-se que a consistência interna $(\alpha=0,74)$ era adequada e muito próxima ao alfa de Cronbach obtido no estudo original. As médias e desvios padrões encontrados na presente amostra (ver Tabela 1) também foram muito próximos aos obtidos no estudo original.

Para analisar as diferenças da autoestima nos quatro grupos de papéis de bullying em relação ao sexo, foi reali- zada uma análise de variância (ANOVA 4X2) com papéis de bullying e sexo como variáveis independentes. A média e o desvio padrão da autoestima nos diferentes papéis de bullying para meninos e meninas são apresentados na Tabela 1.

Tabela1. Média e Desvio Padrão da Autoestima nos Diferentes

\begin{tabular}{lcccc}
\hline & \multicolumn{2}{c}{ Meninas } & \multicolumn{2}{c}{ Meninos } \\
\hline Papéis de bullying & $\mathrm{M}$ & $\mathrm{dp}$ & $\mathrm{M}$ & $\mathrm{Dp}$ \\
Vítimas & 2,84 & 0,35 & 2,78 & 0,45 \\
Agressores & 3,01 & 0,39 & 2,89 & 0,37 \\
Vítimas/agressores & 2,78 & 0,36 & 2,92 & 0,36 \\
Testemunhas & 2,89 & 0,39 & 3,05 & 0,40 \\
\hline
\end{tabular}

Os resultados da ANOVA apontaram uma interação entre sexo e papéis de bullying em relação à autoestima $[F(3,398)=2,95 ; p<0,05]$, como pode ser visto na Figura 1. Devido à interação, foram realizadas análises de variância oneway para o sexo masculino e para o sexo feminino. Estas análises apontaram diferenças significativas entre os papéis de bullying. Testes Post Hoc (Tukey) apontaram que, no grupo de vítimas/agressores, os adolescentes do sexo masculino apresentaram médias de autoestima significativamente superiores em relação às do sexo feminino. Ainda entre os adolescentes do sexo masculino, verificou-se que o grupo de testemunhas apresentou maior média de autoestima que o grupo das vítimas. Em relação ao sexo feminino, verificou-se que o grupo de agressoras apresentou média mais alta de autoestima que o grupo das vítimas/agressoras.

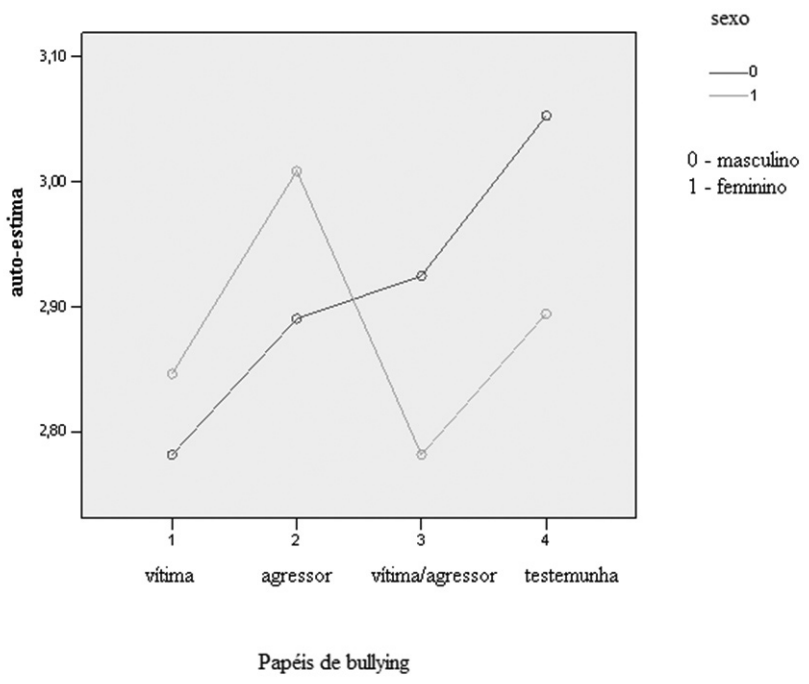

Figura 1. Interação das variáveis autoestima e papéis de bullying por sexo. 


\section{Discussão}

A interação verificada entre sexo e papéis de bullying em relação à autoestima indica que, no grupo vítimas/ agressores, o sexo masculino apresentou média superior de autoestima em relação ao sexo feminino. Entre as meninas, baixos níveis de autoestima estão relacionados com o papel de vítima/agressor, o que não ocorre entre os meninos. Uma explicação para estes achados pode estar na diferença quanto aos fatores que influenciam a autoestima de meninos e meninas. Conforme a literatura (Heatherton \& Wyland, 2003), a autoestima das meninas é fortemente influenciada pelos relacionamentos, enquanto a autoestima dos meninos é influenciada pelo sucesso de seus objetivos. A dimensão social, que inclui o cuidado e a integração interpessoal, parece ser um fator que apresenta grande influência na autoestima das meninas. Para elas, o retorno de pessoas significativas representa um fator de grande importância. As meninas priorizam as emoções e os eventos sociais, passam grande parte do seu tempo trocando informações, segredos e criando intimidade. Durante o período da adolescência, as meninas ficam mais suscetíveis à opinião e aceitação dos pares. As meninas valorizam as amizades mais que os meninos, os quais buscam maior desempenho em atividades como esporte e outros desafios pessoais. Para os meninos, o desempenho pessoal, como, por exemplo, a competição ou o pensamento individual, exerce grande influência na sua autoestima

O grupo vítima/agressor é, dentre os grupos de papéis de bullying, o que possui índices mais altos de rejeição e maltrato pelos colegas (Berger, 2007; Lopes, 2005). Diferenciam-se das vítimas e agressores típicos por serem impopulares e pelo alto índice de rejeição entre seus colegas (Robin e cols., 2005). É plausível que o impacto emocional da rejeição seja maior para as meninas que, enquanto no papel de vítimas/agressoras, são rejeitadas por seus pares. Com a rejeição, passam a ver a si mesmas com desaprovação, percebem-se como incapazes, incompetentes e sem muito valor. Outro agravante que parece funcionar como fator de risco para as meninas durante a adolescência, deixandoas mais vulneráveis aos fatores estressantes do meio e da sociedade, são as mudanças hormonais que ocorrem com a puberdade (Bell e cols., 2005). A puberdade é um período crítico para as meninas, que, diferente de outros períodos do desenvolvimento, apresentam desvantagens em relação aos meninos. A literatura (Bell e cols., 2005) aponta alguns mecanismos que podem explicar estas diferenças de gênero. Tais mecanismos incluem diferentes fatores de risco e proteção, tanto do meio ambiente quanto genéticos, diferentes níveis desses fatores, ou ainda diferentes desafios culturais ou ambientais que meninos e meninas encontram e podem diferenciar o desenvolvimento de algum problema ou dificuldade em particular. Em relação aos meninos, o grupo vítimas/ agressores apresenta níveis mais elevados de autoestima. Ao contrário das meninas, a autoestima dos meninos vítimas/ agressores parece não sofrer grande impacto com a rejeição de seus colegas. É plausível que os meninos sejam menos suscetíveis à aprovação ou desaprovação externa. Para os meninos, o sucesso de seus objetivos pessoais parece representar um fator de maior influência na sua autoestima. É possível que as diferentes exigências culturais juntamente com características pessoais influenciem a forma como o processo de vitimização é experienciado por meninos e meninas. É provável que a rejeição experimentada pelo grupo vítima/agressor cause impacto negativo na autoestima das meninas, que parece ser influenciada em grande parte pelos relacionamentos. Novos estudos são sugeridos a fim de verificar a relação entre rejeição de pares e autoestima em meninos e meninas.

Em relação ao sexo feminino, verificou-se que o grupo de agressoras apresentou média mais alta de autoestima que o grupo de vítimas /agressoras. Dentre as meninas, o grupo de agressoras possui altos níveis de autoestima. Os agressores típicos diferenciam-se das vítimas/agressores pela popularidade. Contrário ao que ocorre no grupo de vítimas/ agressores, o grupo de agressores é bem aceito pelos pares e recebe retorno positivo, fazendo provavelmente com que as meninas agressoras percebam-se como capazes, competentes e com grande valor. O retorno positivo dos pares em relação aos agressores parece influenciar de forma positiva a autoestima das meninas. Estes achados encontram suporte na literatura (Lopes, 2005; Rolim, 2008), que aponta os agressores como figuras populares e bem aceitas pelos colegas. Os próprios agressores veem sua agressividade como qualidade e têm opiniões positivas sobre si mesmos (Lopes, 2005). Sentem prazer e satisfação em dominar, controlar e causar dano aos outros. O agressor pode alcançar um status importante, consolidando sua capacidade de dominar e sua superioridade. Os achados do presente estudo contrariam os resultados encontrados por Rigby e Cox (1996) em um estudo que apontou uma associação entre baixa autoestima e agressores do sexo feminino. Estas diferenças provavelmente ocorreram porque o estudo realizado por Rigby e Cox (1996) não diferenciou o grupo de agressoras puras do grupo de vítimas/agressoras.

Em relação ao sexo masculino, verificou-se que o grupo de testemunhas apresentou maior média de autoestima que o grupo das vítimas. Entre os meninos, baixos níveis de autoestima estão relacionados ao papel de vítima. É plausível que o fato de não possuir recursos, status ou habilidade para se defender, reagir ou cessar o bullying cause impacto na autoestima dos meninos, que passam a se perceber como fracos e sem valor. É possível que a sensação de impotência em se defender dos agressores e cessar o bullying cause um impacto negativo na autoestima dos meninos vitimizados. Estes dados corroboram os achados de Rigby (1998) que, em seus estudos a respeito da personalidade das vítimas, encontrou interação entre escores baixos de autoestima e o papel de vítima.

\section{Considerações finais}

Foi possível verificar que o bullying apresenta diferentes implicações na autoestima de meninas e meninos 
envolvidos nos diferentes papéis e variações de autoestima nos diferentes papéis para o mesmo sexo. Os resultados fornecem dados interessantes no que diz respeito ao bullying e às diferenças entre os sexos. Os trabalhos que visam à prevenção e redução do bullying devem ser estruturados tendo em mente as diferenças entre os sexos. É necessário que se aborde o bullying de maneira diferenciada para cada papel e, em específico, para cada sexo. Sexo e gênero são importantes fontes de variabilidade no comportamento de crianças e adolescentes. Saber que meninos e meninas diferem no padrão de certos tipos de comportamento é o primeiro passo para o conhecimento a respeito dos mecanismos que podem produzir estas diferenças.

Uma vez apontadas diferenças na autoestima de meninos e meninas nos diferentes papéis de bullying, o conhecimento detalhado dos mecanismos subjacentes a cada papel se faz necessário, juntamente com a prevenção dos efeitos deletérios. Estudos futuros deveriam enfocar especificamente o bullying entre as meninas, uma vez que este ainda é pouco investigado. Os dados obtidos através deste estudo visam a enriquecer e ampliar o conhecimento sobre o fenômeno bullying, possibilitando um maior conhecimento acerca dos adolescentes e sua relação com seus pares. Este estudo buscou compreender o bullying como um comportamento agressivo baseado e sustentado por valores culturais do contexto, assim como um modo de relação estabelecido entre pares.

O bullying é um fenômeno presente em praticamente todas as escolas ao redor do mundo (Berger, 2007). Os estudos relacionados ao bullying têm apontado a gravidade das consequências da exposição a abusos frequentes pelos pares. Este fato remete-nos ao sofrimento experienciado por um número expressivo de adolescentes em nossa cultura. A gravidade do fenômeno não pode ser desprezada nas escolas que, por vezes, desconhecem ou minimizam a magnitude de tal fenômeno. A escola desempenha um papel de grande importância no desenvolvimento social de crianças e adolescentes e não pode ser considerada apenas como um espaço destinado à aprendizagem formal ou ao desenvolvimento cognitivo. Portanto, a escola precisa se transformar, adaptar-se à realidade e às demandas culturais atuais e atuar no sentido de prevenir e controlar o bullying, assim como outros comportamentos interativos inadequados e prejudiciais ao desenvolvimento, e não funcionar como um agente mantenedor do sofrimento psicológico dos envolvidos nessas situações.

\section{Referências}

Bandeira, C. M. (2009). Bullying: Auto-Estima e diferenças de gênero. Dissertação de Mestrado, Universidade Federal do Rio Grande do Sul, Porto Alegre.

Bell, D. J., Foster, S. L., \& Mash, E. J. (2005). Understanding behavioral and emotional problems in girls. Em D. J. Bell, S. L. Foster \& E. J. Mash (Orgs.), Handbook of behavioral and emotional problems in girls (pp. 1-21). New York: Plenum Publishers.
Berger, K. S. (2007). Update on bullying at school: Science forgoten? Developmental Review, 27, 90-126.

Cantini, N. (2004). Problematizando o bullying para a realidade brasileira. Tese de Doutorado, Pontifícia Universidade Católica de Campinas, Campinas, São Paulo.

Coopersmith, S. (1989). Coopersmith - self-esteem Inventory. Palo Alto, CA: Consulting Psychologists Press.

Crick, N. R., \& Grotpeter, J. K. (1995). Relational aggression, gender, and social-psychological adjustament. Child Development, 66, 710-722.

Garandeau, C. F., \& Cillessen, A. H. N. (2006). From indirect aggression to invisible aggression: A conceptual view on bullying and peer group manipulation. Aggression and Violent Behavior, 11, $641-654$

Gini, G., \& Pozzoli, T. (2006). The role of masculinity in children's bullying. Sex Roles, 54, 585-588.

Heatherton, T. F., \& Wyland, C. L. (2003). Assessing self-esteem. Em S. J. Lopez \& C. R. Snyder (Orgs.), Positive psychological assessment: a handbook of models and measures (pp.219-233). Washington, DC : American Psychological Association.

Hutz, C. S. (2000). Adaptação brasileira da escala de auto-estima de Rosenberg. Manuscrito não-publicado, Universidade Federal do Rio Grande do Sul, Porto Alegre, Rio Grande do Sul.

Liang, H., Flisher, A. J., \& Lombard, C. J. (2007). Bullying, violence, and risk behavior in South African school students. Child Abuse \& Neglect 31, 161-171.

Lisboa, C. S. M. (2005). Comportamento agressivo, vitimização e relações de amizade em crianças em idade escolar: Fatores de risco e proteção. Tese de Doutorado, Universidade Federal do Rio Grande do Sul, Porto Alegre, Rio Grande do Sul.

Lopes, A. A. N. (2005). Bullying - Comportamento agressivo entre estudantes. Jornal de Pediatria, 81(5), 164-172.

Middelton-Moz, J., \& Zawadski, M. (2007). Bullying - Estratégias de sobrevivência para crianças e adultos (R. C. Costa, Trad.). Porto Alegre: Artmed. (Trabalho original publicado em 2002).

Olweus, D. (1993). Bullying at school. What we know and what we can do. Oxford, UK: Blackwell.

Rigby, K. (1998). The relationship between reported health and involvement in bully/victim problems among male and female secondary school students. Journal of Health Psychology, 3(4), 465-476. 
Rigby, K., \& Cox, I. (1996). The contribution of bullying at school and self-esteem to acts of delinquency among Australian teenagers. Personal and Individual Differences, 21(4), 609-612.

Robin L., Toblina,T., Schwartza, D., Gormanb, A. H., \& Abouezzeddinea, T. (2005). Social-cognitive and behavioral attributes of aggressive victims of bullying. Applied Developmental Psychology, 26, 329-346.

Rolim, M. (2008). Bullying: O pesadelo da escola, um estudo de caso e notas sobre o que fazer. Dissertação de Mestrado, Universidade Federal do Rio Grande do Sul, Porto Alegre, Rio Grande do Sul.
Rosenberg, M. (1989). Society and the adolescent self-image. Princeton: Princeton University Press.

Steinberg, L. (1999). Adolescence. New York: McGraw-Hill.

Vail, K. (2002). How girls hurt. American School Board Journal, 189(8), 14-18.

Recebido em: 21/10/2009

Reformulado em: 19/05/2010

Aprovado em: 01/06/2010

\section{Sobre os autores}

Cláudia de Moraes Bandeira (kkbandeira @hotmail.com)

Universidade Federal do Rio Grande do Sul, Porto Alegre - RS

Claudio Simon Hutz (claudio.hutz@terra.com.br)

Instituo de Psicologia da Universidade Federal do Rio Grande do Sul, Porto Alegre - RS

\section{Correspondência}

Claudio Simon Hutz

Instituto de Psicologia da Universidade Federal do Rio Grande do Sul

Rua Ramiro Barcelos, 2600-Térreo

Bairro Santa Cecília - Porto Alegre - RS

CEP: 90035-003 